Cite this: Sustainable Energy Fuels, $2018,2,2378$

DOI: $10.1039 / c 8 s e 90060 d$

rsc.li/sustainable-energy

\section{Themed issue on perovskite solar cells: research on metal halide perovskite solar cells towards deeper understanding, upscalable fabrication, long-term stability and $\mathrm{Pb}$-free alternatives}

\author{
Nam-Gyu Park, (D) *a Jinsong Huang (ID *bc and Yabing Qi (D) *d
}

Solar cells or photovoltaic (PV) cells are semiconductor devices that convert solar energy into electricity. This technology is already being used to supply clean and renewable energy to society. However, currently installed PV capacity is still relatively low in many countries. Globally, the total generated electricity from PVs constitutes only $\sim 1 \%$ of worldwide electricity demands. ${ }^{1,2}$ Because solar energy is (i) plentiful (e.g., the amount of energy supplied to the earth within one day is sufficient to meet total global energy needs for one year) and (ii) clean, it is often mentioned as a solution to issues related to global warming and the current deficiency of global electricity demands for future society. Currently, siliconbased solar cells represent the dominant PV technology, accounting for $\sim 93 \%$ of total production, in which the shares of mono- and multi-crystalline silicon technology are $\sim 32 \%$ and $\sim 61 \%$, respectively. The market share of all thin-film technologies amounts to $\sim 4.5 \%$ of total

${ }^{a}$ School of Chemical Engineering, Sungkyunkwan University, Suwon 16419, Korea. E-mail: npark@skku. $e d u$

${ }^{b}$ Department of Applied Physical Sciences, University of North Carolina at Chapel Hill, Chapel Hill, NC, USA. E-mail:jhuang@unc.edu

${ }^{c}$ Department of Mechanical and Materials Engineering, University of Nebraska-Lincoln, Lincoln, NE, USA ${ }^{d}$ Energy Materials and Surface Sciences Unit (EMSSU), Okinawa Institute of Science and Technology Graduate University (OIST), Okinawa 904-0495, Japan. E-mail: Yabing.Qi@OIST.jp production: CdTe $(2.3 \%)$, copper indium gallium diselenide or CIGS $(1.9 \%)$, and amorphous-Si $(0.3 \%){ }^{3}$

As summarized in the "Best ResearchCell Efficiencies" chart from the National Renewable Energy Laboratory (NREL), ${ }^{4}$ a large number of alternative PV materials and technologies designated as "Emerging PV" have been developed over the past three decades, with the aim of producing the next generation of solar cells with even lower costs, higher solar energy-to-electricity power conversion efficiencies (PCEs), longer lifetimes, and more environmentally benign. In addition, being flexible, light-weight, and transparent (or semi-transparent) are desirable features for specific applications such as flexible electronics, portable electronics, wearable electronics, building-integrated photovoltaics (BIPV) etc. Among the several types of PV technology, perovskite solar cells (PSCs) based on organic-inorganic lead halides have emerged as a competitive candidate. It has been demonstrated that PSCs can achieve a certified PCE as high as $23.3 \%$, which outperforms multicrystalline $\mathrm{Si}(\mathrm{PCE}=22.3 \%$ ) and is competitive with thin film solar cells of CIGS (22.9\%) and CdTe (22.1\%). ${ }^{4}$ Furthermore, PSCs are low cost and compatible with low-temperature processing, flexible substrates and large-area fabrication, for example, doctor blading, roll-to-roll processing, spray-coating, printing, chemical vapor deposition, and vacuum evaporation. Due to these unprecedented rapid advances, Tsutomu Miyasaka (Toin University of Yokohama, Japan), Nam-Gyu Park (Sungkyunkwan University, Republic of Korea), and Henry Snaith (University of Oxford, United Kingdom) received recognition as the 2017 Citation Laureates by Clarivate Analytics for their discovery and application of perovskite materials to achieve efficient energy conversion.

The outstanding performance (especially in efficiency) achieved for PSCs is a result of the excellent optoelectronic properties of perovskite materials and the proper matching of perovskites with functional material choices of adjacent charge selective contacts and electrodes. However, there is still plenty of room for further improvements in efficiency, stability and scalable manufacturing. The articles collected in this special issue provide up-to-date advances, challenges, and future directions in PSC research for moving this technology forward towards commercialization.

The first research direction is the deepened understanding of PSCs in terms of material science, which has enjoyed tremendous growth. In their perspective article, Yuanyuan Zhou, Ou Chen and coworkers (DOI: 10.1039/ c8se00315g) point out that, accompanying the rapid developments of $3 \mathrm{D}$, perovskite nanocrystals (NCs) have 
recently attracted much attention. In particular, the authors highlight that the integration of perovskite NCs with thin film PSCs holds high promise for applications that have not been fully explored previously, and further research efforts are invited towards building bridges between perovskite NCs and thin-film PSCs (DOI: 10.1039/c8se00315g). The best performing PSCs have complex structures consisting of several heterojunction interfaces: $\mathrm{FTO} / \mathrm{c}-\mathrm{TiO}_{2}+\mathrm{mp}-$ $\mathrm{TiO}_{2} /$ perovskite/organic based $\mathrm{HTL} /$ cathode $(\mathrm{c}=$ compact, $\mathrm{mp}=$ mesoporous and $\mathrm{HTL}=$ hole transport layer).

Research efforts are being made to observe the dynamical processes taking place within complete devices under solar cell operating conditions. In this context, Michael Saliba, Pankaj Yadav and coworkers (DOI: 10.1039/ c8se00280k) employ electrochemical impedance spectroscopy (EIS) to systematically study the influences of wavelength (blue and red) and light intensity (50-100 $\left.\mathrm{mW} \mathrm{cm}^{-2}\right)$ responses in $\mathrm{FTO} / \mathrm{c}-$ $\mathrm{TiO}_{2} / \mathrm{Cs}_{x}\left(\mathrm{FAPbI}_{3}\right)_{0.85}\left(\mathrm{MAPbBr}_{3}\right)_{0.15} /$ spiro$\mathrm{OMeTAD} / \mathrm{Au}$ devices $(\mathrm{FTO}=$ fluorinedoped tin oxide, $\mathrm{FA}=$ formamidinium and $\mathrm{MA}=$ methylammonium). The capacitance-frequency responses under blue and red illumination reveal vital information about charge or ion accumulation and provide insights into interfacial electronic characteristics. It has been shown that blue light has maximum absorption at the surface of the perovskite leading to maximum charge or ion accumulation at the $\mathrm{c}-\mathrm{TiO}_{2} /$ perovskite interface. Red light is absorbed in the bulk of the perovskite leading to a more balanced ion and charge carrier distribution. These fundamental aspects were further associated with hysteresis phenomena in PSCs: when PSCs are subjected to red wavelength, devices exhibit a lower hysteresis, while maximum hysteresis was observed under blue light illumination. This work shows the importance of developing suitable tools to probe dynamical processes at interfaces in PSCs.

The second direction is in response to the grand challenge that the community is already making an effort to address, i.e., the upscaling engineering of PSCs aiming towards the fabrication of modules and panels. Various scalable deposition techniques have been demonstrated for PSCs and module development. These techniques include screen printing, slot-die coating, blade coating, spray coating, inkjet printing, and chemical vapor deposition. Yue $\mathrm{Hu}$, Hongwei Han and coworkers (DOI: $10.1039 / \mathrm{c} 8 \mathrm{se} 00332 \mathrm{~g}$ ) employ the screenprinting-based fabrication of PSCs using a $\mathrm{TiO}_{2} / \mathrm{ZrO}_{2} /$ carbon mesoporous scaffold. The devices based on this printable triple mesoscopic structure were able to generate a certified efficiency of $10.4 \%$ over $100 \mathrm{~cm}^{2}$ with operational stability over 10000 h. Maikel F. A. M. van Hest, Kai Zhu and coworkers (DOI: 10.1039/ c8se00368h) present key aspects of the challenges in controlling perovskite film drying and crystal growth during wet-film coating processes. A stable perovskite precursor ink formulation with a wide processing window of up to 20 minutes between film deposition and antisolvent dipping is shown to be critical for fabricating perovskite films with high quality. The versatility and robustness of this wide wet-film processing window are demonstrated for spin coating, slot-die coating, blade coating, and spraycoating, where fabricated PSCs exhibited comparable performances.

The third direction for PSCs is longterm stability under realistic operation conditions, i.e., continuous light illumination and maximum power point tracking. The stability issues of PSCs have captured a considerable amount of research attention and effort, as evidenced by the largest number of articles in this special issue (DOI: 10.1039/ c8se00358k, $\quad 10.1039 / \mathrm{c} 8 \mathrm{se} 00200 \mathrm{~b}$, 10.1039/c8se00218e, 10.1039/c8se00282g and 10.1039/c8se00250a). Efforts are being made in this direction to understand how PSCs suffer from degradation and which products are generated during degradation. These are important questions to be answered in order to design stable PSCs. In this context, Yanfa Yan and coworkers (DOI: 10.1039/ c8se00358k) provide a time resolved mass spectrometry study of the gas species evolved during the photoinduced degradation of state-of-the-art triplecation (FA/MA/Cs) perovskites. It has been demonstrated that the complete removal of MA and the addition of Cs effectively suppress the release of volatile gases, and the best photostability is obtained with FA/Cs mixed perovskites. In another work by Xingzhong Zhao, Yanfa Yan and coworkers (DOI: 10.1039/ c8se00200b), it is shown that the simultaneous incorporation of lead thiocyanate $\left(\mathrm{Pb}(\mathrm{SCN})_{2}\right)$ additive and $\mathrm{Cs}$ cations enhances perovskite crystallinity and lowers the trap density. PSCs with Cs and SCN additives show improved photostability. The overall degradation within a PSC is not only due to the perovskite layer but also adjacent layers (ETL = electron transport layer, HTL and electrodes), as well as interface related chemical degradation processes. Steve Albrecht, Henk J. Bolink and coworkers (DOI: 10.1039/c8se00218e) have carried out a systematic study on the influences of doped and undoped charge selective transport layers by fabricating 5 different configurations of all dry processed $p-i-n$ planar devices. The performance and stability of planar p-i-n PSCs are significantly enhanced when the doped charge transport layers are replaced by undoped alternatives. The development of encapsulation technology applied in PSCs is another important topic and essential to protect not only PSCs from external environments but also reciprocally to protect the environment from the possible leakage of toxic $\mathrm{Pb}$ from $\mathrm{Pb}$ based PSCs. Philip Schulz and coworkers (DOI: 10.1039/c8se00282g) employ a low temperature $\left(60^{\circ} \mathrm{C}\right)$ atomic layer deposition (ALD) process to deposit a thin conformal layer $(\sim 16 \mathrm{~nm})$ of $\mathrm{Al}_{2} \mathrm{O}_{3}$ on top of PSCs after cathode evaporation. This ALD process does not lead to damage of the PSCs and improves longterm device stability. Michael D. McGehee and coworkers (DOI: 10.1039/ c8se00250a) report the development of a glass-glass encapsulation methodology for PSCs that enables the devices to pass the industry standard IEC 61646 damp heat and thermal cycling tests. These works show that industrially relevant encapsulation techniques have the potential to enable the commercial viability of PSCs in terms of sufficient device stability.

The fourth direction regards the toxicity of $\mathrm{Pb}^{2+}$ in high-performing PSCs, 
which constitutes a major concern for investors, manufacturers and consumers.

Therefore, great effort is being directed towards the development of $\mathrm{Pb}$-free perovskite-based solar cells. As a promising alternative, the engineering of socalled double perovskite materials has been receiving great attention, as reported by Tingli Ma and coworkers (DOI: 10.1039/c8se00154e). Double perovskites are formed by replacing divalent $\mathrm{Pb}^{2+}$ ions by monovalent $\mathrm{B}^{+}$and trivalent $\mathrm{B}^{3+}$ ions in the form of an $\mathrm{A}_{2} \mathrm{~B}^{+} \mathrm{B}^{3+} \mathrm{X}_{6}$ structure. For example, the authors synthesized a new double perovskite material of $\mathrm{Cs}_{2} \mathrm{NaBiI}_{6}$. Currently, Pb-free PSCs still exhibit substantially lower performance compared to Pb-based counterparts. Further research effort is needed to develop new alternative $\mathrm{Pb}$-free perovskites as well as to understand the main factors causing low efficiencies. There have been an increasing number of studies on Sn-Pb-mixed low-bandgap perovskite absorbers, which have opened up new opportunities for developing tandem devices based on solely perovskite materials. In their recent study, Tomas Leitjens, Rohit Prasanna, Michael D. McGehee and coworkers (DOI: 10.1039/c8se00314a) achieve a high current density matching in a twoterminal tandem solar cell device and obtain external quantum efficiencies exceeding $80 \%$ in the near infrared region.

The rapid development of perovskite materials and PSCs, well reflected by the exponential growth in the number of related publications, ${ }^{5}$ provides a vibrant backdrop for the wide spectrum of research topics and directions covered by this themed issue. The community's eagerness towards the quest for "what PSC technology needs the most in the next few years" is expected to produce further important findings that will serve as a strong foundation for the development of future perovskite-based PV technology.

\section{References}

1 S. Kurtz, N. Haegel, R. Sinton and R. Margolis, Nat. Photonics, 2017, 11, 3.

2 C. Breyer, D. Bogdanov, A. Gulagi, A. Aghahosseini, L. S. N. S. Barbosa, O. Koskinen, M. Barasa, U. Caldera, S. Afanasyeva, M. Child, J. Farfan and P. Vainikka, Prog. Photovoltaics, 2017, 25, 727-745.

3 Fraunhofer Institute for Solar Energy Systems ISE, https:// www.ise.fraunhofer.de/en.html, accessed date, September 22nd, 2018.

4 National Renewable Energy Laboratory (NREL), Research Cell Efficiency Records, https:/www.nrel.gov/pv/ assets/images/efficiency-chart.png, accessed date, September 22nd, 2018.

5 Web of Science, Thomson Reuters, https://www.webofknowledge.com/, accessed date, September 23rd, 2018. 